OPEN ACCESS

Edited by:

Sheng Zhang,

Yale University, United States

Reviewed by:

Amir Jaberzadeh,

University of Southern California,

United States

Ting Ma,

Harbin Institute of Technology, China

Meiling Li,

Athinoula A. Martinos Center

for Biomedical Imaging, Harvard

Medical School, United States

${ }^{*}$ Correspondence:

Jifeng Guo

guojifeng2003@163.com

Specialty section:

This article was submitted to

Brain Imaging Methods,

a section of the journal

Frontiers in Neuroscience

Received: 11 October 2018

Accepted: 18 January 2019

Published: 04 February 2019

Citation:

Zhang $K$, Tang $Y$, Meng $L$, Zhu $L$,

Zhou X, Zhao Y, Yan X, Tang B and Guo J (2019) The Effects of SNCA rs894278 on Resting-State Brain

Activity in Parkinson's Disease.

Front. Neurosci. 13:47.

doi: 10.3389/fnins.2019.00047

\section{The Effects of SNCA rs894278 on Resting-State Brain Activity in Parkinson's Disease}

\author{
Kailin Zhang ${ }^{1}$, Yan Tang ${ }^{1,2}$, Li Meng ${ }^{3}$, Liping Zhu ${ }^{3}$, Xiaoting Zhou' ${ }^{1}$, Yuwen Zhao', \\ Xinxiang Yan ${ }^{1}$, Beisha Tang ${ }^{1,4,5,6,7,8}$ and Jifeng Guo ${ }^{1,4,5,6 *}$
}

\begin{abstract}
${ }^{1}$ Department of Neurology, Xiangya Hospital, Central South University, Changsha, China, ${ }^{2}$ School of Information Science and Engineering, Central South University, Changsha, China, ${ }^{3}$ Department of Radiology, Xiangya Hospital, Central South University, Changsha, China, ${ }^{4}$ National Clinical Research Center for Geriatric Disorders, Xiangya Hospital, Changsha, China, ${ }^{5}$ Key Laboratory of Hunan Province in Neurodegenerative Disorders, Central South University, Changsha, China, ${ }^{6}$ Center for Medical Genetics, School of Life Sciences, Central South University, Changsha, China, ${ }^{7}$ Parkinson's Disease Center of Beijing Institute for Brain Disorders, Beijing, China, ${ }^{8}$ Collaborative Innovation Center for Brain Science, Shanghai, China
\end{abstract}

The pathogenesis of Parkinson's disease (PD) is not well established. The rs894278 polymorphism of SNCA has been associated with PD. We performed this study to investigate the relationship between rs894278 and PD status on resting-state brain activity, by analyzing the amplitude of low-frequency fluctuation (ALFF). A total of 81 PD patients and 64 healthy controls were recruited. Disease severity and PD stage were evaluated in PD patients using the unified Parkinson's disease rating scale (UPDRS) and the Hoehn and Yahr $(H Y)$ scale, while the cognitive function of all participants was assessed using the mini-mental state examination (MMSE). All participants were genotyped for the rs894278 SNP and underwent a resting state functional magnetic resonance imaging scan. We found that the ALFF values of PD patients in the lingual gyrus and left caudate were lower than those of HCs; and the ALFF values for the right fusiform of participants with $\mathrm{G}$ allele were lower than those of participants without $G$ allele. And we further revealed higher ALFF values in bilateral fusiform in rs894278$G$ carriers than in rs894278-G non-carriers in the PD group and lower ALFF values in bilateral fusiform in rs894278-G carriers than in rs894278-G non-carriers in the HC group. Our findings show that rs894278 and PD status interactively affect the brain activity of PD patients and HCs, and changes in the brain connectomes may play a key role in the pathogenesis of PD. Thus, our work sheds light on the mechanism underlying PD pathogenesis.

\section{Keywords: Parkinson's disease, SNCA, resting state functional MRI, ALFF, brain activity}

\begin{abstract}
Abbreviations: ALFF, amplitude of low-frequency fluctuation; BOLD, blood oxygen level dependent; BP, binding potential; DPARSF, Data Processing Assistant for Resting-State fMRI software; EPI, echo planar imaging; FWHM, full width at half maximum; GMV, gray matter volume; HCs, healthy controls; HY, Hoehn and Yahr scale; LBs, Lewy bodies; LEDD, levodopa equivalent daily dose; MCI, mild cognitive impairment; MMSE, mini-mental state examination; MNI, Montreal Neurological Institute; PD, Parkinson's disease; PET, positron emission tomography; rs-fMRI, resting-state functional magnetic resonance imaging; SNP, single nucleotide polymorphism; SPM, Statistical Parametric Mapping; TE, echo time; TR, repetition time; UPDRS, unified Parkinson's disease rating scale.
\end{abstract}




\section{INTRODUCTION}

Parkinson's disease is a common neurodegenerative disease that affects $1.7 \%$ of Chinese population over the age of 65 (Zhang et al., 2005). The clinical manifestation of PD involves impairment of normal motor functions, such as bradykinesia, rigidity, resting tremor, and postural instability (Goedert et al., 2013). Additionally, PD is associated with non-motor symptoms that indicate multi-system impairment, such as autonomic dysfunction, sensory dysfunction, sleep disorders, and cognitive impairment (Yang et al., 2016). The hallmark pathology of PD is characterized as the loss of dopaminergic neurons in the substantia nigra pars compacta and neurite terminals projecting to the striatum, as well as the emergence of LBs in the remaining neurons (Dickson, 2012). By the time symptoms manifest enough for patients to seek PD diagnoses, striatal dopamine levels have already been reduced by over 50\% (Rajput et al., 2008).

The etiology of PD remains unclear; however, it is understood that a complex interaction between genetics, environmental factors, and aging are responsible for disease development and progression. Genetic factors remain the focus of current research and, among all of the genetic factors related to PD, only a very small percentage follow monogenic inheritance patterns (Karimi-Moghadam et al., 2018). The remaining polymorphisms either do not directly contribute to PD and work in concert with other genetic variants or explain only a minute portion of disease pathology. In both cases, identified variants maintain the ability to be protective against or contribute to PD pathology (Guo et al., 2015). SNCA, the first gene discovered to have a causative link to PD, is located on 4q22.1 and encodes the protein a-synuclein. A-synuclein is abundant in the brain and is thought to regulate dopamine release and transport, induce fibrosis of the microtubule-associated protein tau, and reduce the reactivity of neurons to various apoptosis stimuli. Furthermore, LBs, important pathological markers of PD, are formed by the abnormal accumulation of a-synuclein (Deng and Yuan, 2014). Mounting evidence implicates genetic variants in SNCA to play an important role in the pathogenesis of PD. Rare mutations in SNCA can cause PD that follows Mendelian inheritance patterns, but this does not appear to be a common cause of PD in the Han population (Deng et al., 2015). However, many common variants of $S N C A$, including rs894278, contribute to PD risk and help to explain the appearance of PD in non-mendelian patterns (Xu W. et al., 2015). The rs894278 SNP was first found to be associated with PD through a genome-wide association study (Satake et al., 2009), and subsequent case-control studies in several independent Han populations have confirmed its impact on PD (Tan et al., 2010; Chang et al., 2011; Liu et al., 2013). Although a strong link between rs894278 and PD has been uncovered, the mechanism governing the impact of rs894278 on PD has yet to be elucidated.

Resting-state functional magnetic resonance imaging is a noninvasive quantitative brain MRI technique that explores the brain's functional activity by analyzing changes in BOLD signals in resting individuals (Fox and Raichle, 2007). This technique has been widely applied in PD research. ALFF analysis was first proposed by Zang et al. (2007), and primarily focuses on the magnitude of low-frequency oscillations associated with hemodynamic activity. Studies of rs-fMRI in PD patients have demonstrated that changes in ALFF values in certain brain regions can effectively distinguish PD patients from normal controls, suggesting that ALFF value may be utilized as a diagnostic biomarker for PD (Skidmore et al., 2013b; Tang et al., 2017a). Furthermore, additional studies have shown that ALFF alteration is also correlated with PD symptoms such as apathy, depression, and PD motor symptoms (Skidmore et al., 2013a; Chen et al., 2015).

Depending on their location and context, genetic variants affects cellular function and in turn can manifest as alteration in normal brain structure and function. When variants present in a manner that influences brain activity, these changes can be reliably measured by fMRI. With this knowledge, a new strategy has been developed that combines genetic polymorphism information with rs-fMRI images to investigate alterations in brain activity and how variants contribute to disease state. This technique is a very sensitive and efficient way to characterize the influence of a particular SNP on brain activity and has previously been applied to neurological and psychological diseases such as schizophrenia (Wei et al., 2013), major depressive disorder (Wang et al., 2017), and Alzheimer's disease (Wang et al., 2014). The sensitivity of this technique enables not only the detection of brain areas affected by associated SNP, but also provides enough resolution to decipher alterations in brain network architecture (Tang et al., 2017b). Information on which networks and how they are altered provides insights into how specific SNPs lead to disease phenotypes and the mechanisms that govern phenotypic changes. A recent study conducted by Nombela et al. (2014) applied this strategy to PD using task fMRI and found that rs4680 of COMT gene, rs9468 of MAPT gene, and rs429358 of $A P O E$ gene modulated different brain areas each associated with different cognitive domains. Additionally, brain function is altered before clinical symptoms present. Thus, the ability to genotype individuals and detect altered brain activity (or fMRI images) characteristic of PD can be used as a powerful tool for early diagnosis before other clinically diagnosable features emerge (van Nuenen et al., 2009).

In this study, we speculate that the genotype of the rs 894278 SNP influences the brain activity of PD patients and is involved in the pathogenesis of PD. We utilize the strategy outlined above to explore the differential brain activity of PD patients and HCs with different SNCA rs894278 genotypes.

\section{MATERIALS AND METHODS}

\section{Participants}

A total of $81 \mathrm{PD}$ patients and 64 age and sex matched HCs were recruited at Xiangya Hospital, Central South University, from April 1, 2013 to February 26, 2017. PD diagnosis was made, according to the UK PD Society Brain Bank clinical diagnostic criteria (Hughes et al., 1992), by two experienced neurologists. All participants were right-handed Chinese individuals without any other organic diseases, mental disorders or contraindications to MRI scanning. In addition, all patients were free from 
antiparkinsonian drugs for at least $12 \mathrm{~h}$ before the clinical and MRI assessments.

This study was approved by the Xiangya Hospital ethics committee, and written informed consent was provided by every participant prior to beginning this study.

\section{Clinical Assessment}

Disease severity and PD stage were evaluated in PD patients using the UPDRS and the HY scale, while the cognitive function of all participants was assessed using the MMSE. The tremor, rigidity, bradykinesia, and posture/gait score were calculated by the sum of scores from item $20+21$, item 22 , item $23+24+25+26+31$, and item $27+28+29+30$ of UPDRS, separately. In addition, the LEDD was computed based on existing methods (Tomlinson et al., 2010), namely: total LEDD $=$ immediate release $\mathrm{L}$-dopa dose $\times 1+$ L-dopa $\times 0.33$ (concurrent entacapone ingestion) + controlled release L-dopa dose $\times 0.75+$ duodopa $\times 1.11+$ pramipexole $\times 100+$ selegiline $\times 10+$ rasagiline $\times 100+$ piribedil $\times 1+$ amantadine $\times 1+$ ropinirole $\times 20+$ rotigotine $\times 30$. MMSE scores between PD patients and HCs were compared, while UPDRS, HY, and LEDD scores were compared between different PD patient groups.

\section{Genotyping}

Genomic DNA of all participants was extracted from peripheral blood samples using standard phenol-chloroform procedures. The SNP rs894278 was genotyped using time-of-flight mass spectrometry. Every participant was either classified as a G carrier or $\mathrm{G}$ non-carrier after genotyping.

\section{MRI Data Acquisition}

MRI data were acquired using a 3.0T GE Signa MR scanner (General Electric, Fairfield, CT, United States) in the Department of Radiology of Xiangya Hospital, Central South University. Resting-state functional images covering the whole brain were recorded using a gradient EPI sequence with the following parameters: $\mathrm{TR}=2,000 \mathrm{~ms}$, $\mathrm{TE}=30 \mathrm{~ms}$, voxel size $=3.44 \times 3.44 \times 4.60$, flip angle $=90^{\circ}$, slice number/thickness $=32 / 4.0 \mathrm{~mm}$. Every participant was asked to rest calmly and keep his or her head still during the MRI scan. During a 6-min time period, 180 time points were obtained for each participant.

\section{MRI Data Preprocessing}

MRI data were preprocessed with the DPARSF ${ }^{1}$, which is based on SPM version $8^{2}$. The procedures used were as follows: From the total of 180 slices of rs-fMRI data from each participant, the first 10 were removed, and the remaining 170 slices were corrected. Head motion of each slice was realigned, while data from participants with head motion exceeding $1.5 \mathrm{~mm}$ or $1.5^{\circ}$ were excluded. The slices were normalized to the MNI template after dimension changes and the quality of resultant images were manually checked. Next, smoothing was carried out using a

${ }^{1}$ http://www.restfmri.net

${ }^{2}$ https://www.fil.ion.ucl.ac.uk/spm/
$4 \times 4 \times 4$ FWHM Gaussian kernel. Following smoothing, linear and quadratic trends resulting from the MRI machine, motion, or noise were removed. Finally, signals of head parameter in six dimensions, global brain, white matter and cerebrospinal fluid were regressed out. After data preprocessing, data from 15 PD patients and $6 \mathrm{HCs}$ were excluded from the study, thus $66 \mathrm{PD}$ patients and $58 \mathrm{HCs}$ remained for the final analyses.

\section{ALFF Analysis}

To remove very-low-frequency drift and high frequency noise, time series between 0.01 and $0.08 \mathrm{~Hz}$ of each voxel were extracted and converted to frequencies using a Fast Fourier Transform and the power spectrum was subsequently obtained. The power spectrum's square root was calculated and is referred to as the ALFF value. The ALFF value of each voxel was then divided by the mean ALFF of the whole brain to produce the mALFF. ALFF values between different groups were compared and significant clusters were obtained and shown using Automated Anatomical Labeling (AAL) brain atlas. Finally, ALFF values of clusters showing significant differences were correlated with clinical assessment scores, such as the UPDRS, UPDRS I, UPDRS II, UPDRS III, UPDRS IV, tremor, rigidity, bradykinesia, and posture/gait scores, HY scale stage, and LEDD, for PD patients who were rs894278-G carriers.

\section{Statistical Analysis}

Demographic and clinical data between PD patients and HCs, as well as clinical data between different groups of PD patients, were analyzed using SPSS version 21.0 (SPSS Inc., Chicago, IL, United States). For continuous variables, analyses were performed using a Student's $t$-test or Mann-Whitney $U$ test. For categorical variables, analyses were performed using $\chi^{2}$ tests. The statistical significance threshold was defined as $P<0.05$.

MRI data between groups were analyzed and compared with SPM8. Full factorial analysis was conducted to analyze the influences of diagnosis and genotype on rs-fMRI images, through which the independent and interactive effects of diagnosis and genotype were obtained in all participants. For areas with a significant diagnosis $\times$ genotype interaction effect, post hoc analyses were subsequently conducted to separately investigate the genotype effect in the PD and HC groups. The statistical significance threshold was defined as $P<0.01$ (AlphaSim corrected). Correlation analysis between ALFF values and clinical data was performed using partial correlation analysis to remove potential confounding MMSE scores (FDR corrected).

\section{RESULTS}

\section{Demographic and Clinical Data}

There were no significant differences in age, sex or genotype between the PD and HC groups. However, the MMSE scores were lower in the PD group than in the HC group (Table 1).

There were no significant differences in the age of onset, course of disease, scores for UPDRS, UPDRS I, UPDRS II, UPDRS III, UPDRS IV, tremor, rigidity, bradykinesia, or 
posture/gait, HY scale stage, or LEDD between rs894278-G carriers and non-carriers among PD patients (Table 2).

\section{Group Based Differences in ALFF}

The full factorial analysis model revealed significant diagnosis effects in the lingual gyrus and left caudate, genotype effects in the right fusiform, and diagnosis $\times$ genotype effects in the right and left fusiform (Table 3 and Figure 1). As depicted in Figure 1, the ALFF values of PD patients in the lingual gyrus and left caudate were lower than those of HCs; and the ALFF values for the right fusiform of participants with $G$ allele were lower than those of participants without $\mathrm{G}$ allele. A post hoc analysis of clusters with

TABLE 1 | Demographic and clinical data between PD and HC groups.

\begin{tabular}{lccc}
\hline Characteristics & PD $(\boldsymbol{n}=\mathbf{6 6})$ & HC $(\boldsymbol{n}=\mathbf{5 8})$ & $\boldsymbol{P}$ \\
\hline Age (years) & $53.76 \pm 10.72$ & $51.67 \pm 10.80$ & 0.284 \\
Sex (male/female) & $35 / 31$ & $29 / 29$ & 0.736 \\
Genotype (G carriers/G non-carriers) & $41 / 25$ & $33 / 25$ & 0.554 \\
MMSE & $26.78 \pm 3.65$ & $27.88 \pm 3.31$ & $0.023^{*}$ \\
UPDRS score & $49.02 \pm 22.05$ & - & - \\
UPDRS I score & $3.00 \pm 1,92$ & - & - \\
UPDRS II score & $13.29 \pm 6.48$ & - & - \\
UPDRS III score & $30.67 \pm 15.26$ & - & - \\
UPDRS IV score & $2.06 \pm 3.22$ & - & - \\
Tremor score & $4.62 \pm 3.62$ & - & - \\
Rigidity score & $5.71 \pm 4.26$ & - & - \\
Bradykinesia score & $13.09 \pm 7.17$ & - & - \\
Posture/gait score & $4.83 \pm 2.89$ & - & - \\
HY scale & $2.33 \pm 0.77$ & - & -
\end{tabular}

Data are shown as the mean \pm standard deviation. "G carriers" represents the participants who possess at least one rs894278 G allele; "G non-carriers" represents the participants who possess no rs894278 G alleles. PD, Parkinson's disease; HC, healthy control; MMSE, mini-mental state examination. ${ }^{*} P<0.05$.

TABLE 2 | Clinical data between rs894278-G carriers and non-carriers among PD patients.

\begin{tabular}{lccc}
\hline Characteristics & G carriers $(\boldsymbol{n}=\mathbf{4 1})$ & G non-carriers $(\boldsymbol{n}=\mathbf{2 5})$ & $\boldsymbol{P}$ \\
\hline Age of onset & $48.37 \pm 12.21$ & $46.72 \pm 11.89$ & 0.547 \\
Course of disease & $5.41 \pm 4.26$ & $7.00 \pm 6.53$ & 0.801 \\
UPDRS score & $47.90 \pm 19.77$ & $50.84 \pm 25.70$ & 0.603 \\
UPDRS I score & $3.20 \pm 1.72$ & $2.68 \pm 2.21$ & 0.133 \\
UPDRS II score & $13.07 \pm 5.70$ & $13.64 \pm 7.71$ & 0.968 \\
UPDRS III score & $29.85 \pm 2.27$ & $32.00 \pm 16.59$ & 0.583 \\
UPDRS IV score & $1.78 \pm 3.21$ & $2.52 \pm 3.25$ & 0.239 \\
Tremor score & $4.22 \pm 3.64$ & $5.28 \pm 3.54$ & 0.193 \\
Rigidity score & $5.54 \pm 4.62$ & $6.00 \pm 3.67$ & 0.672 \\
Bradykinesia score & $12.95 \pm 6.57$ & $13.32 \pm 8.18$ & 0.841 \\
Posture/gait score & $4.71 \pm 2.87$ & $5.04 \pm 2.96$ & 0.557 \\
HY scale & $2.34 \pm 0.69$ & $2.32 \pm 0.90$ & 0.967 \\
LEDD & $421.25 \pm 247.09$ & $391.00 \pm 312.84$ & 0.330 \\
\hline Data are shown as & &
\end{tabular}

Data are shown as the mean \pm standard deviation. "G carriers" represents the participants who possess at least one rs894278 G allele; "G non-carriers" represents the participants who possess no rs894278 G alleles. PD, Parkinson's disease; UPDRS, unified Parkinson's disease rating scale; HY, Hoehn and Yahr; $L E D D$, levodopa equivalent daily dose. significant diagnosis $\times$ genotype effects further revealed higher ALFF values in G carriers than in non-carriers in the PD group and lower ALFF values in G carriers than in non-carriers in the HC group (Figure 2).

\section{Correlation Analysis}

After removing confounding MMSE scores, we found a significant negative correlation between UPDRS score and ALFF values in the lingual gyrus that showed a diagnosis effect $(P=0.049, r=-0.322)$ and a significant negative correlation between UPDRS I score and ALFF values in the lingual gyrus that showed a diagnosis effect $(P=0.028, r=-0.358)$ in PD patients carrying the rs894278 G allele. We also discovered a significant positive correlation between posture/gait scores and ALFF values in the left fusiform that showed a diagnosis $\times$ genotype interaction effect $(P=0.008, r=0.548)$ and a significant positive correlation between HY scale stage and ALFF values in the left fusiform that showed a diagnosis $\times$ genotype interaction effect $(P=0.008, r=0.548)$ in PD patients without $r 8894278 \mathrm{G}$ allele. Unfortunately, we did not find any significant correlation between ALFF values in the brain regions that showed groupbased differences and clinical assessments after multiple test correlation.

\section{DISCUSSION}

This is the first study to investigate the effect of SNCA rs894278 on ALFF in PD patients and HCs. Our results show that the ALFF values of PD patients in the lingual gyrus and the left caudate were lower than those of HCs, and that the ALFF value of participants with $\mathrm{G}$ allele in the right fusiform were lower than those of participants without $G$ allele. We further reveal higher ALFF values in rs894278-G carriers than in rs894278-G noncarriers in the PD group and lower ALFF values in rs894278-G carriers than in rs894278-G non-carriers in the HC group. These findings provide strong evidence to support the notion that SNCA

TABLE 3 | ALFF comparison of rs894278 between the PD and HC groups with different rs894278 genotypes.

\begin{tabular}{|c|c|c|c|c|c|c|}
\hline \multirow[t]{2}{*}{ Regions (AAL) } & \multirow{2}{*}{$\begin{array}{c}\text { Number of } \\
\text { voxels }\end{array}$} & \multicolumn{3}{|c|}{ Peak MNI coordinates } & \multirow[t]{2}{*}{$T$-value } & \multirow[t]{2}{*}{$P$} \\
\hline & & $x$ & $Y$ & $z$ & & \\
\hline \multicolumn{7}{|l|}{ Diagnosis effects } \\
\hline Lingual gyrus & 138 & 3 & -96 & -12 & -5.7379 & $<0.01$ \\
\hline Caudate_L & 129 & -12 & 12 & 3 & -4.9698 & $<0.01$ \\
\hline \multicolumn{7}{|l|}{ Genotype effects } \\
\hline Fusiform_R & 176 & 30 & -63 & -3 & -4.5923 & $<0.01$ \\
\hline \multicolumn{7}{|c|}{$\begin{array}{l}\text { Diagnosis } \times \text { genotype } \\
\text { effects }\end{array}$} \\
\hline Fusiform_R & 121 & 30 & -33 & -24 & 4.485 & $<0.01$ \\
\hline Fusiform_L & 106 & -30 & -39 & -21 & 4.1886 & $<0.01$ \\
\hline
\end{tabular}

The T-value denotes the statistical value of the peak voxel. ALFF, amplitude of lowfrequency fluctuations; PD, Parkinson's disease; HC, healthy control; MNI, Montreal Neurological Institute; $L$, left; $R$, right. 

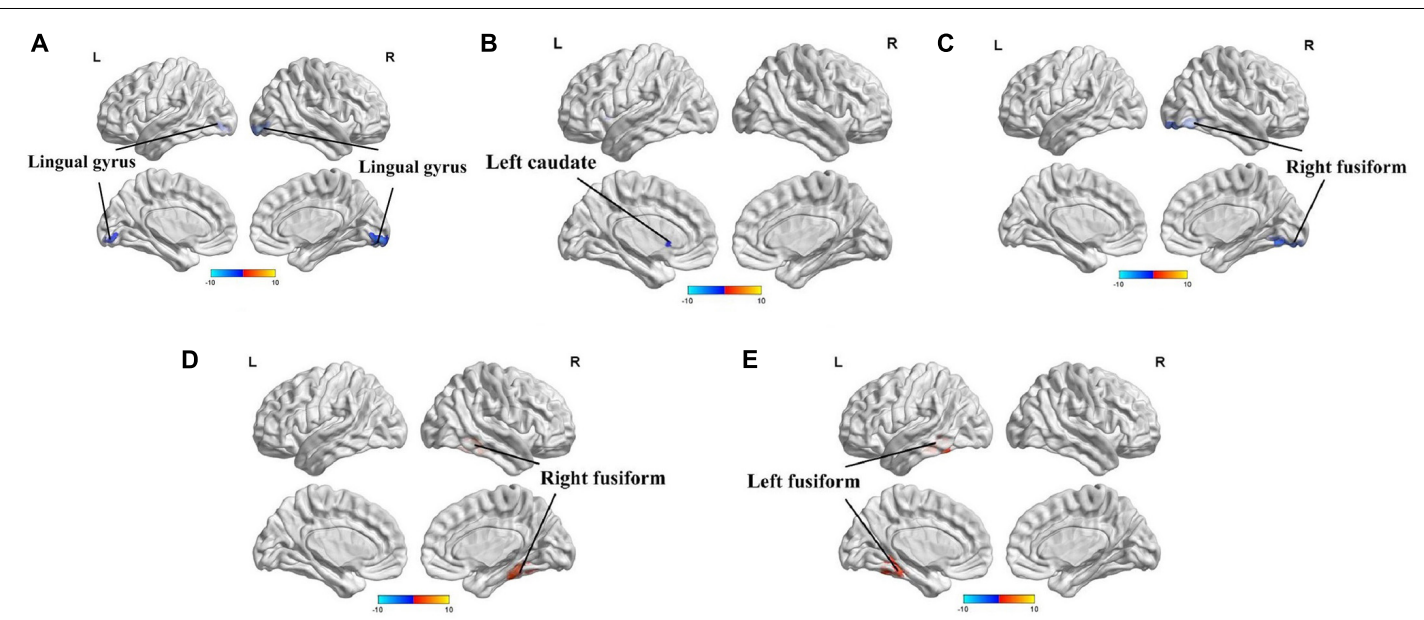

FIGURE 1 | Amplitude of low-frequency fluctuations (ALFF) analysis of resting-state brain activity for G carriers vs. non-carriers of the rs894278 genotype in the Parkinson's disease (PD) and healthy control (HC) groups. (A) ALFF at the lingual gyrus in PD patients is decreased relative to HCs. (B) ALFF at the left caudate in PD patients is decreased relative to HCs. (C) ALFF at the right fusiform in rs894278-G carriers is decreased relative to G non-carriers. (D) ALFF comparisons for the right fusiform in PD patients and HCs for rs894278-G carriers and G non-carriers. (E) ALFF comparisons for the left fusiform in PD patients and HCs for rs894278-G carriers and $\mathrm{G}$ non-carriers.
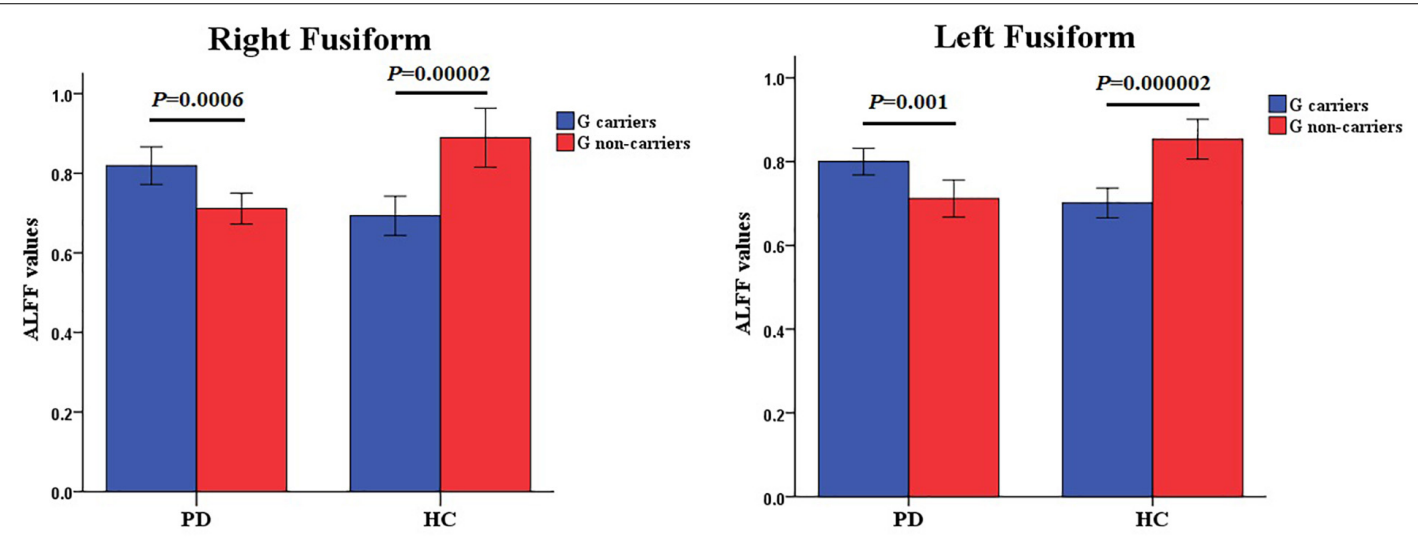

FIGURE 2 | Diagnosis $\times$ genotype effects on ALFF values. The color bar shows post hoc comparisons in the clusters with significant diagnosis $\times$ genotype effects. The differences between rs894278-G carriers and non-carriers in the PD or HC group were significant separately. Data are shown as the mean \pm standard error. ALFF, amplitude of low-frequency fluctuations; PD, Parkinson's disease; HC, healthy control.

rs894278 works in conjunction with PD status to affect brain activity in an interactive manner in PD patients and HCs.

The significant diagnosis $\times$ genotype effect in the bilateral fusiform means that the function of the bilateral fusiform gyrus was influenced by both the diagnosis of PD and the rs894278 genotype. Furthermore, PD patients with the rs894278 G allele had higher spontaneous brain activity than those without the $G$ allele in the bilateral fusiform gyrus, while HCs with the rs894278 $\mathrm{G}$ allele had lower spontaneous brain activity than HCs without the $G$ allele in these regions. This indicates that the presence of a rs $894278 \mathrm{G}$ allele exacerbates PD symptoms though alterations in the fusiform structure and function. The fusiform plays many roles in the proper function in several neurological pathways and contributes to cognitive function, facial recognition, the visual pathway, and autonomic function.
With respect to the ventral visual pathway, irregularities in the fusiform is tightly linked to dysfunction of visual processing and can lead to hallucinations in PD patients (Oishi et al., 2005). Brain GMV in the fusiform of PD patients is smaller than that of HCs, and the decreased GMV in this region is associated with worse cardiovascular autonomic function (Chen et al., 2016). In the fusiform gyrus of PD patients with and without MCI, longitudinal BOLD signals are reduced in patients with MCI relative to those free from cognitive impairment (Ekman et al., 2014). In addition, Rypma et al. (2015) tested the dopamine D1 BP using PET and BOLD signal change via fMRI when the participants were performing a face recognition task; the results showed that the D1 BP and BOLD signal in the fusiform gyrus were significantly related. Taken together, this indicates that the fusiform gyrus affects non-motor functions of PD, and the 
activity of the fusiform can be regulated by dopamine. We found that PD diagnosis and rs894278 genotype can influence fusiform activity, hinting at a link between rs894278 genotype and the non-motor functions of PD. This finding is consistent with a recent study showing that the rs894278 genotype is associated with hyposmia in PD (Li et al., 2017). However, it is difficult to tell whether the dysfunction in the fusiform is caused by PD itself, a compensatory mechanism of the disease, or chronic dopaminergic treatment. Further investigation would be necessary to determine exact underpinnings responsible for fusiform dysfunction in PD patients.

Furthermore, when we divided all participants into two groups according to rs894278 genotype, participants with the rs894278 $\mathrm{G}$ allele had lower brain activity compared to those without the rs894278 G allele in the right fusiform. However, PD patients with the rs894278 $\mathrm{G}$ allele had higher brain activity than those without the rs894278 G allele. Thus, the effect of rs894278 genotype on brain activity is reversed in the PD group. These data indicate that the fusiform may be a key brain region where the rs894278 polymorphism has an impact on PD pathogenesis. As depicted above, the fusiform is associated with cognitive function and therefore the rs894278 SNP is likely associated with the cognitive impairment seem in $\mathrm{PD}$ patients. Prior research has shown that genetic variation of the SNCA gene is associated with dementia in PD (Guella et al., 2016). This previous link between a gene variant and dementia in PD has been characterized, gives weight to our hypothesis that rs894278 SNP is linked to the decline of cognitive function in PD. This evidence indicates that rs894278 is an interesting candidate for future validation with functional studies. Our results also show that PD patients and HCs exhibit different brain activity in the left caudate and lingual gyrus, which are associated with the motor (Tessitore et al., 2012; Xu Y. et al., 2015) and non-motor functions of PD (Niethammer et al., 2013; Jung et al., 2014; Kiferle et al., 2014; Liu et al., 2015), respectively. Thus, we speculate that there is a relationship between the fusiform, caudate, and lingual areas, and the interaction of these regions influences the integrated brain activity of patients and controls. Tan et al. (2011) stated that the fusiform, caudate, and lingual engaged in language learning, and Ulrich and Kiefer (2016) showed that the caudate and ventral visual stream areas (which include the fusiform and lingual gyri) have altered connections in subliminal visuomotor priming. These studies found that the fusiform, caudate and lingual work together in a certain brain activity, which support the idea of the potential relationship between these brain regions.

\section{REFERENCES}

Chang, X. L., Mao, X. Y., Li, H. H., Zhang, J. H., Li, N. N., Burgunder, J. M., et al. (2011). Association of GWAS loci with PD in China. Am. J. Med. Genet. B Neuropsychiatr. Genet. 156b, 334-339. doi: 10.1002/ajmg.b.31167

Chen, H. M., Wang, Z. J., Fang, J. P., Gao, L. Y., Ma, L. Y., Wu, T., et al. (2015). Different patterns of spontaneous brain activity between tremordominant and postural instability/gait difficulty subtypes of Parkinson's disease: a resting-state fMRI study. CNS Neurosci. Ther. 21, 855-866. doi: 10.1111/cns. 12464
Although our findings are robust, there are some limitations to our study. First, cognitive status was not well matched between our PD and HC groups, so results may be affected by interference from regions related to cognitive function. In addition, although the average MMSE scores of the two groups were both in the normal range, the score was slightly lower in the $\mathrm{PD}$ group. This suggests that PD patients may already have had some cognitive impairment even though their MMSE scores showed their cognitive function to be 'normal.'

\section{CONCLUSION}

In conclusion, we investigated the effect of SNCA rs894278 on ALFF in PD patients and HCs and found altered spontaneous activity in certain brain regions that were influenced by PD status and rs894278 genotype. The results indicate that rs894278 affects the occurrence of PD. Furthermore, changes in the brain connectomes may play a key role in the pathogenesis of PD. Overall, our work sheds light on the mechanisms underlying PD pathogenesis.

\section{AUTHOR CONTRIBUTIONS}

KZ, YT, BT, and JG conceived the study. KZ and YT analyzed the fMRI data. LM, LZ, XZ, and YZ collected the fMRI data. LZ, XZ, $\mathrm{YZ}$, and XY collected the clinical data. KZ drafted the manuscript. YT revised the manuscript. BT and JG supervised the study and obtained funding. All authors have approved the final article.

\section{FUNDING}

This work was supported by the National Key Plan for Scientific Research and Development of China (Grant Nos. 2016YFC1306000 and 2017YFC0909101) and the National Natural Science Foundation of China (Grant Nos. 81430023, 81371405, and 81571248).

\section{ACKNOWLEDGMENTS}

The authors thank all the patients and volunteers for their participation in this study. They also thank Dr. Matthew J. W. Armstrong, Department of Human Genetics, Emory University School of Medicine, for language editing.

Chen, M., Lu, C., Chen, P., Tsai, N., Huang, C., Chen, H., et al. (2016). Association between autonomic impairment and structural deficit in Parkinson disease. Medicine 95:e3086. doi: 10.1097/MD.0000000000003086

Deng, H., and Yuan, L. (2014). Genetic variants and animal models in SNCA and Parkinson disease. Ageing Res. Rev. 15, 161-176. doi: 10.1016/j.arr.2014.04.002

Deng, S., Deng, X., Yuan, L., Song, Z., Yang, Z., Xiong, W., et al. (2015). Genetic analysis of SNCA coding mutation in Chinese Han patients with Parkinson disease. Acta Neurol. Belg. 115, 267-271. doi: 10.1007/s13760-014-0347-2

Dickson, D. W. (2012). Parkinson's disease and parkinsonism: neuropathology. Cold Spring Harb. Perspect. Med. 2:a009258. doi: 10.1101/cshperspect.a009258 
Ekman, U., Eriksson, J., Forsgren, L., Domellöf, M., Elgh, E., Lundquist, A., et al. (2014). Longitudinal changes in task-evoked brain responses in Parkinson's disease patients with and without mild cognitive impairment. Front. Neurosci. 8:207. doi: 10.3389/fnins.2014.00207

Fox, M. D., and Raichle, M. E. (2007). Spontaneous fluctuations in brain activity observed with functional magnetic resonance imaging. Nat. Rev. Neurosci. 8, 700-711. doi: 10.1038/nrn2201

Goedert, M., Spillantini, M. G., Del Tredici, K., and Braak, H. (2013). 100 years of Lewy pathology. Nat. Rev. Neurol. 9, 13-24. doi: 10.1038/nrneurol.2012.242

Guella, I., Evans, D. M., Szu-Tu, C., Nosova, E., Bortnick, S. F., Goldman, J. G., et al. (2016). alpha-synuclein genetic variability: a biomarker for dementia in Parkinson disease. Ann. Neurol. 79, 991-999. doi: 10.1002/ana.24664

Guo, J. F., Li, K., Yu, R. L., Sun, Q. Y., Wang, L., Yao, L. Y., et al. (2015). Polygenic determinants of Parkinson's disease in a Chinese population. Neurobiol. Aging 36, 1765.e1-1765.e6. doi: 10.1016/j.neurobiolaging.2014.12.030

Hughes, A. J., Daniel, S. E., Kilford, L., and Lees, A. J. (1992). Accuracy of clinical diagnosis of idiopathic Parkinson's disease: a clinico-pathological study of 100 cases. J. Neurol. Neurosurg. Psychiatry 55, 181-184. doi: 10.1136/jnnp.55.3.181

Jung, J., Kang, J., Won, E., Nam, K., Lee, M., Tae, W., et al. (2014). Impact of lingual gyrus volume on antidepressant response and neurocognitive functions in major depressive disorder: a voxel-based morphometry study. J. Affect. Disord. 169, 179-187. doi: 10.1016/j.jad.2014.08.018

Karimi-Moghadam, A., Charsouei, S., Bell, B., and Jabalameli, M. R. (2018). Parkinson disease from mendelian forms to genetic susceptibility: new molecular insights into the neurodegeneration process. Cell. Mol. Neurobiol. 38, 1153-1178. doi: 10.1007/s10571-018-0587-4

Kiferle, L., Ceravolo, R., Giuntini, M., Linsalata, G., Puccini, G., Volterrani, D., et al. (2014). Caudate dopaminergic denervation and visual hallucinations: evidence from a ${ }^{123} \mathrm{HI}$-FP-CIT SPECT study. Parkinsonism Relat. Disord. 20, 761-765. doi: 10.1016/j.parkreldis.2014.04.006

Li, Y., Kang, W., Zhang, L., Zhou, L., Niu, M., and Liu, J. (2017). Hyposmia is associated with RBD for PD patients with variants of SNCA. Front. Aging Neurosci. 9:303. doi: 10.3389/fnagi.2017.00303

Liu, C., Ma, X., Song, L., Fan, J., Wang, W., Lv, X., et al. (2015). Abnormal spontaneous neural activity in the anterior insular and anterior cingulate cortices in anxious depression. Behav. Brain Res. 281, 339-347. doi: 10.1016/ j.bbr.2014.11.047

Liu, J., Xiao, Q., Wang, Y., Xu, Z. M., Wang, Y., Yang, Q., et al. (2013). Analysis of genome-wide association study-linked loci in Parkinson's disease of Mainland China. Mov. Disord. 28, 1892-1895. doi: 10.1002/mds.25599

Niethammer, M., Tang, C. C., Ma, Y., Mattis, P. J., Ko, J. H., Dhawan, V., et al. (2013). Parkinson's disease cognitive network correlates with caudate dopamine. Neuroimage 78, 204-209. doi: 10.1016/j.neuroimage.2013.03.070

Nombela, C., Rowe, J. B., Winder-Rhodes, S. E., Hampshire, A., Owen, A. M., Breen, D. P., et al. (2014). Genetic impact on cognition and brain function in newly diagnosed Parkinson's disease: ICICLE-PD study. Brain 137(Pt 10), 2743-2758. doi: 10.1093/brain/awu201

Oishi, N., Udaka, F., Kameyama, M., Sawamoto, N., Hashikawa, K., and Fukuyama, H. (2005). Regional cerebral blood flow in Parkinson disease with nonpsychotic visual hallucinations. Neurology 65, 1708-1715. doi: 10.1212/01. wnl.0000187116.13370.e0

Rajput, A. H., Sitte, H. H., Rajput, A., Fenton, M. E., Pifl, C., and Hornykiewicz, O. (2008). Globus pallidus dopamine and Parkinson motor subtypes: clinical and brain biochemical correlation. Neurology 70(16 Pt 2), 1403-1410. doi: 10.1212/ 01.wnl.0000285082.18969.3a

Rypma, B., Fischer, H., Rieckmann, A., Hubbard, N., Nyberg, L., and Bäckman, L. (2015). Dopamine D1 binding potential predicts fusiform BOLD activity during face-recognition performance. J. Neurosci. 35, 14702-14707. doi: 10.1523/ JNEUROSCI.1298-15.2015

Satake, W., Nakabayashi, Y., Mizuta, I., Hirota, Y., Ito, C., Kubo, M., et al. (2009). Genome-wide association study identifies common variants at four loci as genetic risk factors for Parkinson's disease. Nat. Genet. 41, 1303-1307. doi: 10.1038/ng.485

Skidmore, F. M., Yang, M., Baxter, L., von Deneen, K., Collingwood, J., He, G., et al. (2013a). Apathy, depression, and motor symptoms have distinct and separable resting activity patterns in idiopathic Parkinson disease. Neuroimage 81, 484-495. doi: 10.1016/j.neuroimage.2011.07.012

Skidmore, F. M., Yang, M., Baxter, L., von Deneen, K. M., Collingwood, J., He, G., et al. (2013b). Reliability analysis of the resting state can sensitively and specifically identify the presence of Parkinson disease. Neuroimage 75, 249-261. doi: 10.1016/j.neuroimage.2011.06.056

Tan, E. K., Kwok, H. H., Tan, L. C., Zhao, W. T., Prakash, K. M., Au, W. L., et al. (2010). Analysis of GWAS-linked loci in Parkinson disease reaffirms PARK16 as a susceptibility locus. Neurology 75, 508-512. doi: 10.1212/WNL. 0b013e3181eccfcd

Tan, L. H., Chen, L., Yip, V., Chan, A. H., Yang, J., Gao, J. H., et al. (2011). Activity levels in the left hemisphere caudate-fusiform circuit predict how well a second language will be learned. Proc. Natl. Acad. Sci. U.S.A. 108, 2540-2544. doi: 10.1073/pnas.0909623108

Tang, Y., Meng, L., Wan, C. M., Liu, Z. H., Liao, W. H., Yan, X. X., et al. (2017a). Identifying the presence of Parkinson's disease using low-frequency fluctuations in BOLD signals. Neurosci. Lett. 645, 1-6. doi: 10.1016/j.neulet.2017.02.056

Tang, Y., Xiao, X., Xie, H., Wan, C. M., Meng, L., Liu, Z. H., et al. (2017b). Altered functional brain connectomes between sporadic and familial Parkinson's patients. Front. Neuroanat. 11:99. doi: 10.3389/fnana.2017.00099

Tessitore, A., Amboni, M., Cirillo, G., Corbo, D., Picillo, M., Russo, A., et al. (2012). Regional gray matter atrophy in patients with Parkinson disease and freezing of gait. AJNR Am. J. Neuroradiol. 33, 1804-1809. doi: 10.3174/ajnr.A3066

Tomlinson, C. L., Stowe, R., Patel, S., Rick, C., Gray, R., and Clarke, C. E. (2010). Systematic review of levodopa dose equivalency reporting in Parkinson's disease. Mov. Disord. 25, 2649-2653. doi: 10.1002/mds.23429

Ulrich, M., and Kiefer, M. (2016). The neural signature of subliminal visuomotor priming: brain activity and functional connectivity profiles. Cereb. Cortex 26, 2471-2482. doi: 10.1093/cercor/bhv070

van Nuenen, B. F., van Eimeren, T., van der Vegt, J. P., Buhmann, C., Klein, C., Bloem, B. R., et al. (2009). Mapping preclinical compensation in Parkinson's disease: an imaging genomics approach. Mov. Disord. 24(Suppl. 2), S703-S710. doi: $10.1002 / \mathrm{mds} .22635$

Wang, L., Day, J., Roe, C. M., Brier, M. R., Thomas, J. B., Benzinger, T. L., et al. (2014). The effect of APOE epsilon4 allele on cholinesterase inhibitors in patients with Alzheimer disease: evaluation of the feasibility of resting state functional connectivity magnetic resonance imaging. Alzheimer Dis. Assoc. Disord. 28, 122-127. doi: 10.1097/WAD.0b013e318299d096

Wang, Y., Li, L., Xu, C., Cao, X., Liu, Z., Sun, N., et al. (2017). Polymorphism of ERK/PTPRR genes in major depressive disorder at resting-state brain function. Dev. Neuropsychol. 42, 231-240. doi: 10.1080/87565641.2017.1306527

Wei, Q., Kang, Z., Diao, F., Guidon, A., Wu, X., Zheng, L., et al. (2013). No association of ZNF804A rs1344706 with white matter integrity in schizophrenia: a tract-based spatial statistics study. Neurosci. Lett. 532, 64-69. doi: 10.1016/j.neulet.2012.10.062

$\mathrm{Xu}$, W., Tan, L., and Yu, J. T. (2015). The link between the SNCA gene and parkinsonism. Neurobiol. Aging 36, 1505-1518. doi: 10.1016/j.neurobiolaging. 2014.10.042

Xu, Y., Wei, X., Liu, X., Liao, J., Lin, J., Zhu, C., et al. (2015). Low cerebral glucose metabolism: a potential predictor for the severity of vascular parkinsonism and Parkinson's disease. Aging Dis. 6, 426-436. doi: 10.14336/AD.2015. 0204

Yang, Y., Tang, B. S., and Guo, J. F. (2016). Parkinson's disease and cognitive impairment. Parkinsons Dis. 2016:6734678. doi: 10.1155/2016/673 4678

Zang, Y. F., He, Y., Zhu, C. Z., Cao, Q. J., Sui, M. Q., Liang, M., et al. (2007). Altered baseline brain activity in children with $\mathrm{ADHD}$ revealed by resting-state functional MRI. Brain Dev. 29, 83-91. doi: 10.1016/j.braindev.2006.07.002

Zhang, Z. X., Roman, G. C., Hong, Z., Wu, C. B., Qu, Q. M., Huang, J. B., et al. (2005). Parkinson's disease in China: prevalence in Beijing, Xian, and Shanghai. Lancet 365, 595-597. doi: 10.1016/s0140-6736(05)17909-4

Conflict of Interest Statement: The authors declare that the research was conducted in the absence of any commercial or financial relationships that could be construed as a potential conflict of interest.

Copyright (c) 2019 Zhang, Tang, Meng, Zhu, Zhou, Zhao, Yan, Tang and Guo. This is an open-access article distributed under the terms of the Creative Commons Attribution License (CC BY). The use, distribution or reproduction in other forums is permitted, provided the original author(s) and the copyright owner(s) are credited and that the original publication in this journal is cited, in accordance with accepted academic practice. No use, distribution or reproduction is permitted which does not comply with these terms. 